\title{
LA PREHISTORIA DEL MODO TRIBUTARIO DE PRODUCCIÓN
}

por

\author{
J. M. VICENT GARCÍA \\ Centro de Estudios Históticos C.S.I.C.
}

RESUMEN: El trabajo discute algunas implicaciones analiticas de las propuestas de Haldon acerca del "modo tributario de producción" como categoria bistónica general, capaz de describir la forma de las relaciones sociales predominantes en las formaciones sociales pre-capitalistas. Desde el momento en que asumimos esta tesis, cada uno de los procesos particulares de emergencia de formaciones tributarias "primarias» debe ser considerado como un caso particular de un evento general: el origen de la sociedad de clases. A pesar de la evidente centralidad de esta cuestión para la Teoria Social, ba recibido muy poca atención en los debates.

Se presenta el esbozo un marco teórico general para abordar este problema, a través de la discusión del concepto de "Revolución Neolitica", entendido en términos sociales. La conclusión más importante de esta discusión es que el umodo tributario de producción» no deriva directamente del "comunismo primitivo». La Arqueologia y la Antropologia nos muestran una rica veriedad de formas sociales en las cuales es posible reconocer uformas parentales de extracción del excedente». Podremos bablar, por lo tanto, de formas pre-clasistas de explotación. La discusión sobre este punto nos conduce finalmente a proponer la recuperación de conceptos como "sociedad germánica" y "comunidad asiática" para dar cuenta de esta variedad.

Palabras Clave: Preshistoria, Revolución Neolítica, sociedades primitivas, modos de producción pre-capitalistas, origen del Estado.

ABSTRACT: The paper discusses some analytbical implications of Haldon's proposals concerning the concept of utributary mode of production» as a general historical catbegory describing the predominant form of social relations in pre-capitalist social formations. Since we assume this thesis, individual processes of emergence of epristine» tributary social formations must be seen as particular cases of a general event: the origin of class society. That event has raised not attention in general controversy in despite of its evident centrality for Social Theory.

The paper presents the outline of a general theoretical framework in order to tackle the problem, through the discussion of the concept of "Neolitbic Revolutions, understood in social terms. The main conclusion of sucb discussion is

Hiipanis, LVIII/3, núm. 200 (1998) 823-839 
that the tributary mode of production didn't proceed directly from uprimitive communism». Archeology and Anthropology show us a rich variety of social forms in wich we can recognize "kin-ordered forms of surplus extraction", that is, pre-classist forms of exploitation. This discussion leads us finaly to propose the convenience of recovering concepts such as "rgermanic society" and "asiatic community" to account for this variety.

KEY WORDS: Prehistory, Neolithic Revolution, primitive society, precapitalist modes of production, origin of State.

\section{INTRODUCCIÓN}

Mi propósito en estas páginas es representar el punto de vista de un prehistoriador en el debate sobre el «modo tributario de producción» (a partir de ahora MTP). Este punto de vista es el resultado de mi propia experiencia en el campo de la Edad del Bronce en la Península Ibérica y de los debates que su interpretación histórica ha generado en los últimos años. No obstante, creo que de esta experiencia se pueden extraer elementos generalizables que pueden tener valor en el debate teórico sobre los modos de producción pre-capitalistas. Trataré de poner de manifiesto estos elementos evitando la referencia a cuestiones concretas de arqueología prehistórica.

La mayoría de los especialistas que se ocupan de las formaciones sociales prehistóricas se identifican a si mismos como arqueólogos, queriendo con ello significar que el tipo de problemas de investigación que les ocupan pasan en todo caso por la interpretación del registro arqueológico en términos de categorias sociológicas o históricas, pero no por intervenir en la definición o crítica de las mismas. En este sentido, y más allá de lo que estas páginas puedan aportar al problema concreto del MTP como categotía histórica, si alguna tesis quiero defender, es la necesidad de acabar con la exclusión de la Prehistoria del gran debate teórico de la Historia y las Ciencias sociales.

Es conocida la atención que dedicaron Marx y Engels a informarse sobre lo que en su época se sabía sobre las llamadas «sociedades primitivas» y el papel determinante que esos pocos conocimientos llegaron a desempeñar en su visión global de la Historia. Por ello sorprende que más de un siglo después, un siglo a lo largo del cuál nuestro conocimiento sobre las etapas iniciales del desarrollo de la Humanjiad ha experimentado un crecimiento exponencial, los herederos de aquellos pensadores y sus críticos acostumbren a prestar tan poca atención a esa masa de conocimientos. No es cuestión de discutir aquí los motivos de esta situación, en los que las peculiares pretensiones epistemológicas de la Arqueologia tienen sin duda un papel destacado, sino de señalar las ventajas de su superación. Estas ventajas serian patentes para la propia Atqueologia, condenada en la actualidad a ser un campo para «segundas lecturas» del pensamiento histórico y sociológico, y por lo tanto a una fundamental irrelevancia intelectual. Pero no debían serlo menos para el conjunto de las Ciencias Sociales y el pensamiento histórico, que probablemente ganarían algo al someter a conside-

Hi.pania, IVIII/3, núm. 200 (1998) 823-839 
ración la masa de conocimiento sobre las formas originarias de la sociabilidad humana que la Arqueologia ofrece.

Desde mi punto de vista, esta perspectiva arqueológica permite plantear «en el límite» algunas de las cuestiones centrales del debate. La idea del MTP como categoría globalizadora que puede dar cuenta de todos los modos de producción precapitalistas ha surgido y se ha desarrollado en contextos, como las polémicas sobre el origen del capitalismo, la teoría de la dependencia, o los estudios comparativos entre formaciones «feudales», en los que rara vez se plantea la cuestión de lo que podemos llamar «límite inferion» de aplicabilidad de la categoría. En la mayor parte de los casos en los que ha parecido pertinente discutir la caracterización de una formación social como «tributaria» estamos ante sociedades con Estado, cuyo proceso de formación se remite a formaciones descriptibles, a su vez, como "tributarias" y «estatales».

El problema que quiero plantear es el que surge al considerar lo que podemos llamar estados tributarios primarios, es decir, aquellos cuya formación no puede retrotraerse a la disolución o transformación de sociedades tributarias precedentes y/o sus estados. En tales casos, y antes de cualquier análisis, nos encontramos con procesos de transición que proceden directamente a partir de formaciones sociales descriptibles como "primitivas» (en un sentido que enseguida discutiremos), carentes, por supuesto, de instituciones estatales y, en principio, de relaciones sociales que quepa describir como «clasistas». Es decir, el problema ya no es explicar la génesis de un estado, una estructura determinada de clases o una configuración específica de la forma tributaria de apropiación del excedente, sino del Estado, de la sociedad de clases, de la propia forma tributaria de apropiación del excedente. De aceptar las propuestas en discusión sobre el MTP como «modo de producción precapitalista» único, y por lo tanto otorgarle rango de categoría histórica general, como de hecho propone Haldon ${ }^{1}$, debemos poder encontrar en su definición argumentos suficientes para dar cuenta de todos los procesos de génesis de sociedades tributarias en términos de rasgos comunes y analíticamente relevantes. Dicho en otros términos, debemos poder afrontar la cuestión del origen del MTP con la misma claridad analítica con la que, desde Marx, se afronta lá cuestión del origen del Capitalismo.

Las evidentes dificultades para hacer semejante cosa marcan una dirección interesante para explorar las limitaciones y ambigüedades de la formulación en discusión y las de la propia noción de umodo de producción» en cuanto categoría histórica. Estas dificultades proceden, por una parte, de la gran variedad de procesos históricos concretos que pueden ser descritos como «transiciones a la sociedad tributaria» y/o «formación de estados tributarios originarios»; por otra estriban en la falta de categorías que permitan abordar la consideración analítica de las formaciones pre-tributarias. ¿Cuál es el modo de producción del

\footnotetext{
1993.

1 Hal.DON, John, The State and the Tributary Mode of Production, Verso, London-New York,
} 
que el MTP es la resolución dialéctica como el Capitalismo lo es del MTP? ¿Existen formas pre-clasistas de explotación, o el MTP sucede directamente al "comunismo primitivo»?

Ante la imposibilidad de desarrollar todas las cuestiones que se derivan de estas preguntas, me limitaré en las páginas que siguen a discutir preliminarmente dos problemas generales implícitos en ellas: (1) la relación entre la Revolución Neolítica y el surgimiento del MTP y (2) la naturaleza de las formas pre-clasistas de apropiación del excedente, en relación con el reconocimiento de modos de producción pre-tributarios.

\section{LA REVOLUCIÓN NEOLITICA Y EL MODO TRIBUTAARIO DE PRODUCCIÓN}

Existe una práctica unanimidad en considerar que el precedente histórico del MTP en cuanto modo de producción predominante en las sociedades de clases pre-capitalistas, es un modo de producción "comunitario", que corresponde a una sociedad «sin clases». La mayoría de los autores que han considerado el problema, empezando por el propio Marx, han dado por buena esta generalización. Sin embargo, semejante afirmación deja de lado la gran variedad de formas de organización social que preceden a la aparición de las primeras sociedades clasistas, y que ahora conocemos gracias al desarrollo sistemático de la Arqueología prehistórica. Difícilmente podemos conformarnos, en un análisis más preciso que quisiera dar cuenta de estos resultados, con términos tan vagos como «sociedad igualitaria» o «modo de producción comunitarion, para casos tan distintos como las sociedades de cazadores del $\mathrm{Pa}$ leolítico Superior y las comunidades campesinas de la Edad del Bronce europea, en las que los rasgos de desigualdad afloran de manera evidente. Resulta significativa a este respecto, aunque no la vamos a discutir aquí, la ambigüedad con la que la expresión "comunismo primitivo" ha sido utilizada en la propia tradición marxista.

La alusión a la importancia de la Revolución Neolítica salva con frecuencia esta insuficiencia. Debemos suponer que la Revolución Neolítica, es decir, el proceso a través del cuál estas formaciones sociales "primitivas» e «igualitarias», basadas en economías de caza-recolección (según los términos consensuales en Prehistoria) devienen en sociedades agrarias incursas universalmente en procesos de creciente diferenciación social, es, en si misma, la matriz de constitución primaria del MPT.

Tres de los principales proponentes de la formulación MTP, Amin, Haldon y Wolf ${ }^{2}$, constituyen una excepción en el mencionado desinterés general por incluir la prehistoria y las sociedades llamadas "primitivas» en el debate, y han dedicado atención a la Revolución Neolítica en estos términos. Centraré mis comentarios en la propuesta de Haldon.

\footnotetext{
2 Amin, SAmIR, Unequal development, Hassoks, 1976. Haldon, op. cat.. Wolf, Eric R., Europe
} and the People Without History, University of California Press, Berkeley, 1982.

Hippuia, LVIII/3, núm. 200 (1998) 823-839 
Como señala Amin, y recoge Haldon 3 , la Revolución Neolítica establece las posibilidades materiales de surgimiento del MTP, al dar lugar al desarrollo de las fuerzas productivas al que corresponde y por el que es determinada, la estructura fundamental de clases que le es esencial. Según Haldon 4:

«... Most ancient societies wich had moved out of the stage of kinship-based or "primitive communals exploitation (whether urban-centred or not) and begun to develop a class structure founded upon the differential relationships of various social-economic groups to the means of production and distribution, can be understood, at least partially, through the concept of the tributary/feudal mode of production..."

Este «movimiento» se produce mediante la progresiva sustitución en su función de relaciones sociales de producción de las relaciones de parentesco, por relaciones de clase ${ }^{5}$ :

«... tributaty relations of production always necessarily exist in, or generate, state or state-like political-economic formations (...) The transformation of kin- or lineage based modes of surplus appropriation, with only a limited degree of internal economic, but still kin-based, differentiation, into those based clearly on class exploitation, invariably involves the loosening of kinship ties and dissolution of associated form of social praxis"

Cuando esta "pérdida» se produce (por ejemplo mediante la extensión de las redes de poder coercitivo más allá de los límites del parentesco \%, tesulta inevitable la generación de formaciones económico-políticas de carácter estatal. Haldon advierte que estos procesos dependen fuertemente de sus propios factores históricos y culturales específicos, y que, en el caso de que se deban a factores endógenos y no sean impuestos por asimilación o conquista, son, a menudo, muy graduales ?.

Estos argumentos proveen, de un marco adecuado para abordar las sociedades arqueológicas que se desartollan entre el comienzo del Neolítico y las primeras formaciones estatales en la mayor parte del mundo. No quiero discutir aquí, por lo tanto, los propios argumentos, sino polemizar con una posible interpretación evolucionista de los mismos que, a mi entender, se manifiesta en un cierto finalismo implícito en la última observación de Haldon citada, sobre "causas endógenas".

Llamo "finalismo» a la suposición implícita de que todas las formaciones sociales resultantes de la Revolución Neolítica son sociedades tributarias in nuce, en las que inevitablemente las formas de extracción del excedente basadas en el parentesco irán siendo sustituidas por relaciones de clase basadas en el

3 HALDON, op.cit., pág, 65.

4 Ibidem, pág. 87.

5 Ibidem, pág. 88

6 Ibidem.

7 Ibidem, pág. 89. 
acceso diferencial a los recursos. Sin duda esto es así en un cierto número de casos, pero esto no nos autoriza a extraer ninguna conclusión respecto a la inevitabilidad y universalidad del fenómeno. Por otra parte, la suposición de que el estado tributario es la conclusión inevitable de la Revolución Neolítica nos eximiría de la necesidad de explicar lo que es específico de cada proceso de formación de Estado, y es precisamente esto lo que puede dar valor al concepto como herramienta en el caso de la Prehistoria reciente.

Efectivamente, desde el punto de vista de la experiencia de los prehistoriadores, y probablemente de los antropólogos, la situación es bien distinta: aparentemente, sólo un pequeño número de las formaciones sociales que alcanzan el grado de desarrollo de las fuerzas productivas que llamamos «neolítico» han emprendido el camino de la sustitución de las relaciones de parentesco como forma predominante de las relaciones de producción por relaciones de clase como un proceso «endógeno». Incluso en las zonas clásicas de "origen del Estado» (Mesopotamia, el Valle del Indo, China, Mesoamérica, área andina) la emergencia de formaciones estatales está separada por un lapso de dos o tres milenios del comienzo de la agricultura.

Puede decirse, por lo tanto, que entre la Revolución Neolítica y la Revolución Industrial la mayor parte de la Humanidad ha vivido bajo formas parentales de organización social. Hay que decir también que frecuentemente esto ha sido así incluso para formaciones sociales preclasistas que hayan quedado sujetas a alguna forma de dependencia tributaria con entidades estatales, aunque lo normal es que en estos casos los procesos de formación endógena de clases se inicien o vean reforzados.

Así pues, los procesos de formación endógena de clases son históricamente excepcionales, aunque, dada su fuerza expansiva, resultan más «visibles». Esto desplaza la carga de la prueba hacia el campo de esos procesos: si la formación endógena de clases no es una tendencia inevitable de todas las sociedades agrarias, entonces requiere una explicación en cada caso. Tal explicación debe consistir en establecer bajo qué condiciones las relaciones de clase, tan extrañas a una sociedad ordenada por el parentesco, pudieron llegar a romper un tipo de organización social tan estable y capaz de resistir el empuje de los factores de cambio.

Volviendo al marco teórico esbozado en los textos de Haldon citados más arriba, quisieta centrar la discusión en dos de sus principales tesis explícitas:

1) La comunidad campesina autosuficiente es el resultado de la Revolución Neolítica y la condición de todos los modos de producción precapitalistas.

2) Existen formas de apropiación del excedente en las sociedades «ordenadas por el parentesco». Podemos hablar, por lo tanto, de formas de explotación pre-clasistas.

En la siguiente sección discutiré la primera de estas tesis. Los resultados de esta discusión nos permitirán abordar la segunda en la última sección del trabajo.

Hispanio, LVIII/3, núm. 200 (1998) 823-839 


\section{ASPECTOS SOCIALES DE LA REVOLUCIÓN NEOLÍTICA}

La Revolución Neolítica no es un mero proceso tecno-económico, a través del cuál la comunidad campesina autosuficiente se hace materialmente posible mediante la adquisición de las técnicas de producción de alimentos. El desarrollo de las fuerzas productivas que se deriva de la domesticación de plantas y animales determina y es determinado por un cambio radical en la estructura de las sociedades pre-neolíticas. El sentido de ese cambio es hacer socialmente posible la comunidad campesina autosuficiente, algo muy distinto a la práctica de la agricultura oportunista, mediante la transformación de la tierra de objeto de trabajo en medio de producioón ${ }^{8}$ y el establecimiento de la unidad entre los trabajadores y sus condiciones de producción, precisamente en la forma de la producción familiar a pequeña escala 9 .

Por lo que sabemos, las instituciones de las comunidades de cazadoresrecolectores pre-neolíticos se aproximan a lo que los antropólogos evolucionistas tipifican como «sociedades de bandas»: comunidades basadas en formas de parentesco clasificatorio, cuyos efectivos se reclutan por sistemas más o menos restrictivos de agregación que dan acceso a la participación colectiva en los recursos del grupo. El problema básico de las relaciones intergrupales proviene de la necesidad de mantener un grado suficiente de reciprocidad con las comunidades vecinas con objeto de acceder a ayuda exterior en caso de crisis, para compensat la baja productividad del trabajo, la dificultad de constituir reservas y la imposibilidad de controlar los recursos.

Como ha señalado Gilman ${ }^{10}$ esta dependencia de la reciprocidad externa hace inviable cualquier intento de apropiación excluyente de la tierra, que sólo puede tener estatuto de res nullius, reduciendo la propiedad al ámbito de efectos personales individuales que, por otra parte, pueden ser reproducidos por la mayor parte de los miembros de la comunidad sin grandes esfuerzos. Esto impide la eclosión de formas permanentes de desigualdad, al permitir la fisión del grupo como solución inmediata a cualquier conflicto interno. Por lo tanto la forma en la que se realiza la unidad de los seres humanos y sus condiciones de producción es a través del libre acceso generalizado a los recursos y a los productos del trabajo.

Debe ser analíticamente evidente que bajo estos supuestos es inviable la formación de comunidades campesinas en el sentido estricto. La transformación de la tierra en medio de producción agraria se verifica mediante la inversión de trabajo de rendimiento diferido y la constitución de reservas, y esto

8 Marx, K., El Capital. Crítica de la Economía Politica., Fondo de Cultura Económica, México D.F.Versión española de W. Roces. 20 ed., 130 reimpr., 1978 (tres volúmenes) Vol. 1, págs. 131 ss. 113.

9 Marx en Elster, Jon, Making Sense of Marx, Cambridge University Press, 1994, pág.

10 Gilman, Antonio, "Como valorar los sistemas de propiedad a partir de datos arqueológicos», Trabajos de Prebistoria, 54 (2), 1997, pág. 218.

Hispania, LVIII/3, núm 200 (1998) 823-839 
requiere la detracción de una y otras de las condiciones de libre acceso, es decir: su apropiación. Ahora bien, los sistemas de producción basados en estos supuestos proporcionan un elevado grado de control y predecibilidad de los recursos y aumentan la productividad del trabajo. Estos efectos redundan a su vez en un debilitamiento de la presión sobre la ayuda externa y abren la posibilidad de introducir e institucionalizar mayores grados de exclusividad en la apropiación de los recursos. Podemos, por lo tanto, considerar la contradicción entre estas dos formas de obtener seguridad a largo plazo (alianzas externas vs. Intensificación agratia) como la clave analítica del proceso de formación de sociedades campesinas en el Neolítico.

En efecto, los datos arqueológicos sobre el origen y temprana progresión de las prácticas productivas, muestran que la propia domesticación de animales y plantas se produce en el contexto de estrategias de estabilización y control de la producción. El éxito de algunas de estas estrategias, y su efecto multiplicador, llegan a hacer más rentable, en ciertos casos, la elección final de una dependencia crítica de las prácticas agrarias (y la consiguiente instauración de restricciones al uso externo de los recursos) que el mantenimiento de la dependencia crítica de las alianzas intergrupales. En tal caso, el énfasis sobre estas últimas se debe invertir para encontrar mecanismos de bloqueo del acceso externo a recursos propios.

Estos mecanismos, sean cuales fueren, contradicen los principios básicos del orden social precedente, puesto que suponen de hecho el establecimiento de algún principio de exclusividad en la apropiación de la tierra que actuará a nivel intergrupal como «reciprocidad negativas. La transformación de la propiedad comunitaria de la tierra en relación social fundamental que podemos definir como "apropiación patrimonial», es, en esencia, el eje de todo el proceso. Las comunidades que optaron a la larga por una estrategia de dependencia crítica de una agricultura intensiva tuvieron que dotarse de un marco de categorías sociales que les permitiera no sólo presentar la apropiación patrimonial de los recursos y reservas como socialmente legítima, sino incluso "pensarlas.

Por lo que sabemos sobre las sociedades sin estado, también llamadas por los antropólogos uordenadas por el parentesco» (kin ordered), la disolución del orden de bandas debió producirse mediante una transformación en los sistemas de parentesco. En otro lugar ${ }^{11}$ he propuesto la interpretación de C. Meillasoux ${ }^{12}$ sobre la dicotomía entre "parentesco clasificatorio" y "parentesco genealógico" como marco conceptual adecuado para comprender este proceso. El principio de filiación es al mismo tiempo una forma de limitar el acceso a la condición de miembro del grupo, que sólo puede obtenerse por descendencia o matrimonio, y de resolver el problema de la transmisión de la

11 VICENT, Juan, «El Neolítico. Transformaciones sociales y económicas», Boletín de Antropologia Americana, 24, 1991, págs. 31-61. XXI. 1987

12 Mellasoux, Claude, Mujeres, Graneros y Capitales. Economía doméstica y Capitalismo. Siglo

Higania, LVIII/3, núm $200(1998)$ 823-839 
propiedad sobre los medios de producción considerados como "patrimonio». Por otra parte, la organización interna de la comunidad sobre principios genealógicos abre la posibilidad de introducir grados legítimos de insolidaridad en el interior de la misma al ir desplazando al linaje los límites de la reciprocidad generalizada que antes eran coextensivos a todo el agregado de familias que constituye el grupo. Esto implica, a largo plazo que la contradicción entre apropiación comunitaria y solidaridad intergrupal se desplaza al interior mismo de la comunidad. Más adelante discutiremos el papel de esta contradicción en el surgimiento de formas de desigualdad y explotación.

Es decir que la Revolución Neolítica no puede ser reducida a un proceso de desarrollo de las fuerzas productivas, sino que es una auténtica «revolución» en el sentido técnico del término en la tradición marxista: un proceso de morfogénesis social resultante de las contradicciones entre el desartollo de las fuerzas productivas y las relaciones sociales de producción. Y si se me permite seguir jugando con la vieja y querida terminología, entre esta Revolución Neolítica y las formaciones tributarias nos haría falta otra «sevolución» que la creencia en el carácter inmanente de la explotación de clase en las sociedades post-neolíticas impide conceptualizar y aislar como proceso histórico.

Esta es la posición analítica que corresponde a la «Revolución Urbana» de Gordon Childe ${ }^{13}$, un concepto propuesto precisamente para dar cuenta de la formación de los estados arcaicos, y que tal vez pudiera ser rehabilitado en el contexto del presente debate. En cualquier caso, el problema que quiero discutir surge al considerar simultáneamente los procesos «urban-centred or not» ${ }^{14}$, desde la óptica de una categoría globalizadora como umodo de produccióñ). Los procesos de génesis del Estado en las tempranas sociedades urbanas han centrado la atención de los investigadores ${ }^{15} \mathrm{y}$ representan el patrón clásico al que puede aplicarse sin problemas el marco trazado por Haldon. Sin embargo, como hemos visto, esta vía hacia el MTP es claramente excepcion. En la mayor parte de los casos nos encontramos con que la implantación de la Revolución Neolítica no desemboca en la Revolución Urbana, sino en la formación de sociedades agrarias segmentarias, muy estables a largo plazo.

Por último, de acuerdo con los registros arqueológico y etnográfico, puede decirse que en la mayor parte de estas sociedades segmentarias post-neolíticas y pre-tributarias existen rasgos más o menos acusados de diferenciación social, que nos remiten a formas no comunitarias de apropiación del excedente, aparentemente contenidas dentro de las relaciones de parentesco. Podemos, por lo tanto, plantear la existencia, o al menos la necesidad analítica, de «kin- or line-

13 CHILDE, Vere Gordon, "The Urban Revolution", en Mark P. Leone (ed.), Contemporary Arcbaeology. A Guide to Theory and Contributions, Southern Illinois University Press, London \& Amsterdam, 1972, págs. 43-51.

14 HALDON, op.cit., pág. 87.

15 Vid. Manzanilla, Linda, «Early Utban Societies: Challenges and Perspectives», en L. Manzanilla (ed.), Emergence and Cbange in Early Urban Societies, Fundamental Issues in Archaelogy, Plenum Press, New York-London, 1997, págs. 3-39.

Hispania, LVIII/3, núm. 200 (1998) 823-839 
age modes of surplus appropriation» y tal vez la pertinencia de considerar un modo de producción pre-tributario distinto del «comunab». En cualquier caso, ambas cosas implicarían alguna forma de «explotación preclasista». En la sección siguiente se discute esta cuestión más detalladamente.

\section{FORMAS PRE-TRIBUTARIAS DE EXPLOTACION}

La tesis sobre la existencia de formas de explotación pre-clasistas puede resultar incluso escandalosa desde ciertas ortodoxias marxistas. No es mi propósito, sin embargo, argumentar aquí a favor de esta tesis, que se da por sentada en la discusión, sino, como se dijo al principio, discutir su posible interpretación evolucionista en relación con el problema del surgimiento del MTP.

De acuerdo con la discusión anterior, el «modo parental de extracción del excedente» no es un mero episodio transicional, teleológicamente comprensible como una relación tributaria incipiente, sino la forma predominante de las relaciones sociales post-neolíticas durante la mayor parte de la Historia. Como tal modo de producción, se opone tanto al MTP como al teórico umodo comunal primitivon ${ }^{16}$. Es decir: la emergencia de relaciones tributarias no es una «evolución» lógica de las relaciones parentales de desigualdad, un cambio de la escala de la explotación, sino un tipo de relación cualitativamente distinta que contradice al marco social vigente, del mismo modo que éste contradice el de la sociedad preneolítica, en la que tales formas de desigualdad eran lógica y ontológicamente imposibles. Dicho en otros términos: el MTP no es la negación del "comunismo primitivo", sino la negación de su negación.

En primer término, es necesario aclarar en qué sentido podemos decir que la forma tributaria de apropiación del excedente no es una evolución lógica de las formas basadas en el parentesco, sino un tipo de relación cualitativamente distinta y contradictoria con ella. Esto nos debe permitir abordar el problema de la pertinencia de considerar uno o varios «modos de producción pre-tributarios», como de hecho hace $\mathrm{E}$. Wolf ${ }^{17}$ con su «kin-ordered mode of productions.

Siguiendo a Wolf ${ }^{18}$ hay que empezar por poner en cuestión la propia importancia del parentesco en el orden social primitivo. Lo que realmente quiere decir el que estas sociedades estén "ordenadas por el parentesco» no es que las relaciones sociales se reduzcan a las determinadas por un sistema de parentesco, sino que todas las relaciones sociales posibles se expresan bajo las categorías del parentesco, que, de esta manera, sobredetermina todas las prácticas sociales. Una sociedad ordenada por el parentesco es aquella en la que toda relación social se establece en términos de «teciprocidad» ${ }^{19}$. De este modo, la

16 HaLdon, Op. cit., pág. 66.

17 Wolf, op. cit., págs. 88 y ss.

18 Ibidem, pág. 89.

19 SAHLIns, Marshall, Las Sociedades Tribales, Labor, Barcelona, 1984.

Hispania, LVIIJ/3, núm. 200 (1998) 823-839 
unidad social básica se define por los límites del círculo de reciprocidad generalizada marcado por una unidad de parentesco, real o ficticio, que constituye un «segmento» autónomo. Pero esto no debe ocultar el hecho de que este principio de segmentación responde a unas determinaciones materiales que hacen de la comunidad campesina local autosuficiente la unidad básica de producción y reproducción social, Este hecho material se expresa socialmente como unidad de parentesco precisamente a la escala en la que la reciprocidad generalizada es funcionalmente necesaria para esos fines: organización de la producción y distribución, resolución de conflictos, etc. Del mismo modo, las relaciones de intercambio equilibrado o desigual, dependencia o subordinación económica entre segmentos se expresan en términos de parentesco mítico (descendencia de ancestros comunes), pactos, alianzas, intercambios de tegalos, apoyo militar, venganza de sangre, leyes de hospitalidad, prácticas de exogamia, etc.

El parentesco, así entendido, puede vehicular relaciones de explotación, como un poco a regañadientes admite Haldon ${ }^{20}$ cuya especificidad es que se producen dentro de severas constricciones que provienen de dos fuentes:

1) para ser socialmente aceptables las relaciones de explotación deben darse bajo la forma propia de las relaciones de parentesco, es decir, como relaciones de reciprocidad equilibrada: el excedente no circula como tributo, sino como «don» que satisface la obligación de reciprocidad generada por otro «don» recibido (lo cuál no impide que, en la mayor parte de los casos, ese equilibrio sea puramente ficticio).

2) el sujeto de la apropiación de los medios de producción no es el individuo, sino el segmento, corresponda este al linaje u otra unidad de pertenencia del mismo orden, de tal modo que el criterio de acceso a aquéllos viene dado por la condición de pertenencia a dicha unidad; sin duda esto no impide en muchos casos la posesión efectiva de los recursos por parte de grupos más reducidos o incluso individuos, pero, en última instancia, el marco de legitimidad que da acceso a tal posesión, se remite a la ideología del parentesco.

Estas condiciones han sido admirablemente sintetizadas por Marx, en su análisis de las formaciones clánicas tal como le eran conocidas por las tradiciones escocesas:

«Every one of the usages and traditions of Scottisch gaels reposes upon the supposition that the members of the clan belong to one and the same family. The "great man", the chieftain of the clan, is in one hand quite as arbitrary, on the other quite as confined in his power, by consanguinity, etc., as every father of a family. To the clan, to the family, belonged the district where it had established itself (...) Thus the district was the common propoerty of the familyn ${ }^{21}$

20) HALDON, op. cit., págs. 88-89.

21 MArX, en Bottomore, T.B. y RuBEL, M., KarlMarx Selected Writings in Sociology and Soctial Pbilosopby, C.A. Watts \& Co. Ltd., Londres, 1963, pág. 120. Este texto pertenece al artículo publicado por Marx en el New York Daily el 9 de febrero de 1853, bajo el título "The Duchess of Sutherland and Slaveryn. Agradezco a Pedro Díaz del Rio el conocimiento de este infrecuente texto de Marx. 
En consecuencia, las telaciones de explotación no pueden proceder, al menos formalmente, de la diferente posición de los grupos sociales en relación con los medios de producción, puesto que no hay formalmente otros «grupos sociales» que los definidos por el parentesco. La apropiación del excedente, en este contexto social, se produce a través de la manipulación de los derechos y obligaciones de reciprocidad que definen el tejido de las relaciones sociales, y al mismo tiempo es garantizada por estos mismos derechos y obligaciones:

«(...) Imposts were insignificant, more a tribute by wich the supremacy of «great man" and of his officers was acknowledged, than a rent of land in modern sense, or a source of revenues 22

En el exterior del círculo de reciprocidad «familians la única forma de apropiación de excedente concebible es el saqueo, que en ningún caso puede transformarse por causas «endógenas» en relación de producción.

En el texto citado, Marx describe «a form of social existence wich, in the scale of historical development, stands a full degree below the feudal state; viz. The patriarchal state of societys ${ }^{23}$.

Lo específico, pues, del «modo parental de extracción del excedente» es precisamente su carácter interno con respecto a la comunidad de parentesco. Aquí estriba la principal diferencia analítica con el modo tributatio, lo que le sitúa "un grado por debajo del estado feudal» en cuanto "modo de explotación», aunque si atendemos al otro sentido de la expresión «modo de producción» (la forma de organización de la producción) esas diferencias se hacen muy relativas. En efecto, la mayor parte de las formaciones tributarias originarias (y una parte de las más avanzadas) son, desde este punto de vista de la organización de la producción, el resultado de la agregación de unidades domésticas de producción, superordenadas en estructuras más amplias de dependencia a través de las cuales se produce la extracción de sus excedentes. Por lo tanto, las relaciones de explotación se dan en un marco externo a éllas. El problema de la formación de relaciones tributarias estriba en cómo imponer este marco a las unidades domésticas sin amenazar su reproducción (es decir: como pasar del saqueo al tributo). Este problema tiene dos vertientes: una social, garantizar la reproducción de la unidad doméstica de producción como unidad de los productores y sus condiciones de producción; otra técnica: el excedente debe poder ser materialmente extraido. Más adelante volveremos sobre estas dos condiciones. En cualquier caso, el MTP no es una forma de organización de la producción, sino el resultado de la imposición a las unidades domésticas de producción de un marco superorgánico de desigualdad: el Estado.

Debe haber quedado claro en la discusión precedente el sentido en el que es posible hablar de formas pretributarias de apropiación del excedente, su diferencia con las formas específicamente tributarias y la discontinuidad y oposi-

22 Ibidem, pág. 121 .

23 Ibidem, pág. 120.

Hipania, LVIII/3, núm. 200 (1998) 823-839 
ción que existe entre ambos tipos de explotación. Centraremos ahora la discusión en la forma en que ambos modos de explotación se articulan históricamente para dar forma al «evento central en el desarrollo de la Humanidad: la ruptura ocurrida con la emergencia de la sociedad clasistas ${ }^{24}$.

El modo patrimonial de explotación es propio de sociedades sin Estado, "primitivas» en el sentido que da Pierre Clastres ${ }^{25}$ al término: indivisas, carentes de categorías para integrar la explotación de clase y, en general, protegidas por sus instituciones contra élla: «sociedades contra el Estado» ${ }^{26}$, pero no necesariamente "contra la explotación», siempre que esta no amenace el marco de legitimidad parental.

En efecto, como señala Clastres, estas sociedades no son igualitarias. En ellas hay una distribución asimétrica del poder que inevitablemente genera una distribución asimétrica del excedente comunitario. Pero en última instancia, el poder pertenece a todo el cuerpo social de una manera efectiva, que se manifiesta en un mecanismo de resistencia cuyo funcionamiento podemos reconocer casi universalmente entre las sociedades segmentarias que conocemos: cuando las exigencias de un jefe sobrepasan lo razonablemente exigible a sus seguidores, estos proceden a abandonatle o matarle ${ }^{27}$.

El modo patrimonial de explotación presenta, por lo tanto, una contradicción fundamental: la que existe entre la lógica de la acumulación de excedente, que se apoya en la propia forma de apropiación patrimonial que fundamenta la comunidad campesina autosuficiente, y la de la forma parental de las relaciones sociales, que se traduce en la resistencia de la sociedad al surgimiento de divisiones en su seno. La profundidad de esta contradicción proviene del hecho de que sus dos elementos emanan igualmente de la apropiación patrimonial de los medios de producción como relación social fundamental.

Por lo tanto, cualquier intento de consolidat endógenamente formas de dominación al margen de las relaciones de parentesco pasa por la disposición de medios para bloquear estos mecanismos de autodefensa de la sociedad por parte de los aspirantes a la constitución de una clase dominante. $Y$ estos medios, en principio, no pueden ser otros que un uso efectivo de la coerción y/o la manipulación ideológica. Pero ninguno de estos medios puede ser eficiente si no se apoya en la creación de vínculos de dependencia objetiva (material) entre campesinos y grupos dominantes, vínculos que se reproduzcan a sí mismos al margen de las obligaciones del parentesco, e incluso en contra de éllas.

En efecto, la violencia o la manipulación ideológica (dos formas de «coacción extraeconómicay), o combinaciones de ambos factores y otros, puede crear situaciones de facto en las que la apropiación de excedente se produzca más allá de las obligaciones del parentesco. Pero para que la relación resultante pueda ser considerada una forma de renta esta situación de facto debe presen-

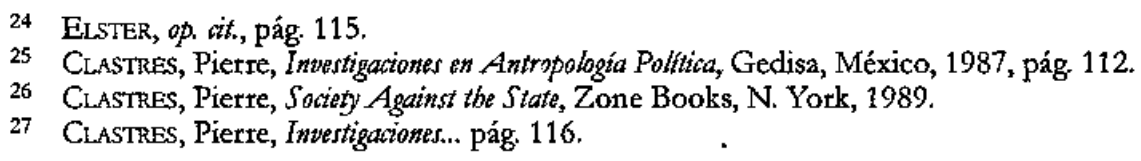


tarse como socialmente aceptable y legitima en términos tales que impliquen su propia reproducción, más allá del alcance físico de la violencia y de la magnitud real de la dependencia objetiva.

La forma más general en la que esta transformación puede producirse es desplazando la legitimidad en la apropiación de los medios de producción desde la unidad parental al grupo dominante. Este desplazamiento es de naturaleza fundamentalmente ideológica y, por lo que sabemos acerca de diversos procesos históricos de formación de estados arcaicos, suele tomar la forma de sistemas religiosos organizados (como en los sistemas templo-palaciales clásicos que dan lugar a los primeros estados dinásticos en el Próximo Oriente, Mediterráneo oriental y otros lugares) y/o (dideologías heróicas» (como las aristocracias agrarias ibéricas de la segunda Edad del Hierro ${ }^{28}$ y sus equivalente en diversos lugares de Europa y el Mediterráneo). Pero lo que, en última instancia, es común a estas dos variantes de ideología tributaria es que se trata de formas de legitimación de estrategias de creación de «dependencia objetiva» entre productores primarios y «tomadores de excedente».

En efecto, al margen de las formas históricas específicas que tome la disolución del orden social parental, la condición general de todo el proceso estriba en lo que hemos llamado «dependencia objetiva». En última instancia, los mecanismos de autodefensa de la comunidad primitiva se bloquean cuando el coste del abandono o la eliminación del jefe excesivamente ambicioso o la resistencia a sus coacciones es mayor que el «coste de la sumisión»" ${ }^{29}$.

Gilman ${ }^{30}$ ha mostrado en sus análisis de un caso paradigmático, la Edad del Bronce en el Sureste de España, cómo «la vulnerabilidad del campesino a la explotación» funciona como condición del desarrollo de relaciones de explotación, y es directamente proporcional al coste en trabajo inmovilizado en los medios de producción, e inversamente a la disponibilidad de recursos equivalentes. Esto explica que las tendencias más intensas hacia la diferenciación social en el conjunto de la Edad del Bronce ibérica estén relacionados con procesos de intensificación agraria. La necesidad de los campesinos de garantizar su acceso a sus inversiones productivas acumuladas facilita típicamente estrategias de creación de dependencia basadas en el intercambio de uprotección a pequeña escala» por excedente. En efecto, la formación de "protection rackets»" está detrás de un gran número de «so-

28 Ruiz, Arturo y Molinos, Manuel, Los Iberos. Análisis arqueologico de un proceso bistórico, Crítica, Barcelona, 1993.

29 HaAs, Jonathan, The Evolution of the Prehistoric State, Columbia University Press, New York, 1982, pág. 158.

30 GiLman, Antonio: «El análisis de clase en la prehistoria del Sureste», Trabajos de Prehistoria, 44, 1987, pp. 27-34; «Unequal development in Copper Age Iberia», en E.M. Brumfiel y T. Eatle (eds.), Specialization, Exchange and Complex Societies, Cambridge University Press, Cambridge, 1987, pp. 22-29; «Como valorar los sistemas de propiedad a partir de datos arqueológicos», Trabajos de Prebistoria, 54 (2), 1997, pp. 81-92.

31 ElsTER, op.cit., pág.167.

1 Hispania, LVIII/3, nưm. 200 (1998) 823-839 
ciedades heroicas», en las que la inestabilidad y la violencia intercomunitaria (frecuentemente más virtual que real) son la condición de relaciones de explotación intracomunitaria, al favorecer la emergencia de grupos dominantes de guerreros, mutuamente interesados en mantenerla a un nivel suficiente como para hacer razonables sus exigencias.

Diversos autores ${ }^{32}$ en distintos campos de investigación han llamado la atención sobre el concepto marxiano de "sociedad germánica» para dar cuenta de estas formaciones agratias situadas justo «un grado por debajo» dei feudalismo, hasta el punto de que han sido a menudo descritas como «feudalismo a peq̨ueña escala». Sin embargo, interesa recalcar que esa diferencia de grado entre el «gran hombre» germánico y el señor feudal, no es (o al menos no sólo) de escala, sino cualitativa: el primero depende crucialmente de la reproducción de las condiciones parentales que definen su posición; el segundo apoya su poder en las condiciones, externas a la propia constitución de la comunidad, que definen el Estado. De hecho una de las características de las formaciones germánicas es precisamente su resistencia al estado ${ }^{33}$.

Las formaciones germánicas surgen sobre la base de la imposición de estrategias de dependencia a campesinos organizados en explotaciones familiares a pequeña escala. El concepto da cuenta de un elevado número de formaciones sociales prehistóricas, históricas y etnográficas ${ }^{34}$ pero no agota la variabilidad de formas de disolución de la sociedad parental. En el otro extremo de la variedad de los procesos de formación de clases encontramos otros tipo de estrategias de creación de dependencia, basadas en el debilitamiento de la autonomía y autosuficiencia de las comunidades campesinas. Estas estrategias tratarian de desarticular la resistencia de estas comunidades incorporándolas en sistemas redistributivos, a menudo apoyados en la creación de infraestructuras de producción supracomunitarias. Tales estrategias invertirían la tendencia al individualismo ideológico propio de los sistemas germánicos, para enfatizar los componentes comunalistas propios de las sociedades parentales, orientándolos hacia formas de acumulación de excedentes fuera del control de las unidades de producción. Este tipo de estrategias están en la base de formación de los sistemas templo-palaciales que ya se han mencionado más arriba, y nos remiten a la intuición de Marx, al situar la dicotomía entre «comunidad asiática» y «ex-

32 MLLER, W.I, Bllodtaking and Peacemaking. Feud, Law, and Society in Saga Iceland, Uiniversity of Chicago Press, Chicago, 1990. Durrembercer, E. Paul, «Property, State, and Self-Destruction in Medieval Iceland", en Robert C. HUnT y Antonio Gilman (eds.), Property in Economic Context, Monographs in Economic Anthropology, 14, University Press of Ametica, LanhamNew York-Oxford, 1998, 171-186. GiLman, Antonio «Ptehistoric European Chiefdoms. Rethinking 'Germanic' Societies", en T. Douglas Price y Gary M. Feinman (eds.), Foundations of Social Inequality, Plenum Press, New York, 1995.

$33 \mathrm{Vid}$ por ejemplo el caso de la sociedad islandesa entre los ss.IX y XIII en los textos de Durrenberger y Miller citados en la nota anterior.

34 Ver una amplia enumeración de ejemplos en Gilman, "Prehistoric European Chiefdoms. Rethinking "Germanic" Societies", cit en nota 32. 
plotación familiar a pequeña escalay en la base del desarrollo histórico de la desigualdad social ${ }^{35}$.

Los procesos de formación endógena de sociedades clasistas, y en general los posibles modos de producción pretributarios, se desarrollarían pues en un espectro delimitado por los extremos del individualismo egermánico» y el comunalismo «asiático». Pero lo que interesa destacar ahora es que esta variedad histórica se remite a un proceso único (analíticamente hablando), que podemos resumir en los siguientes términos: a diferencia del cazador-recolector que goza de una amplia autonomía para reproducir sus medios de subsistencia sin contar con reservas o infraestructuras inmóviles, el campesino depende crucialmente de la acumulación previa de trabajo social necesario para la transformación de la tierra de objeto de trabajo en medio de producción ${ }^{36}$. Este proceso de acumulación es, por lo tanto, la condición general de cualquier forma de apropiación del excedente, tanto porque define la unulnerabilidad del campesino", como hemos visto, cuanto porque es la propia condición material de la existencia de un excedente susceptible de apropiación. Por otra parte, este excedente (sea producto $o$ trabajo) debe ser acumulable y suficiente: la productividad del trabajo campesino (en gran parte dependiente de factores técnicos y de la masa de trabajo social «capitalizado" en los medios de producción) debe permitir la reproducción del grupo explotador al margen de los procesos de producción primaria. Para que los «tomadores de excedente» se puedan constituir y reproducir como clase deben contar con medios para acumular el trabajo o el producto excedente de los campesinos y sustraerlo de los circuitos de reciprocidad parental hasta un nivel suficiente como para eximirles de la participación directa en la producción.

En otro lugar ${ }^{37}$ he propuesto la expresión «acumulación originaria tributaria» para definir este proceso simétricamente a la "acumulación originaria capitalista» de Marx. La simetría se justifica porque es a través de la «acumulación originaria tributarias como se realiza la forma de unidad de los productores con los medios de producción que caracteriza a todas las formaciones precapitalistas y que sólo será disuelta durante el proceso descrito por Marx ${ }^{38}$. Todas las instituciones de las sociedades pre-capitalistas giran de alguna manera en torno a la forma de garantizar esta unidad salvando al mismo tiempo los intereses sociales hegemónicos.

Como se ha visto, este proceso depende básicamente de condiciones materiales que hagan posible la extracción y acumulación del excedente campesino a un nivel suficiente como para rebasar la escala de la ureproducción simples de la comunidad patrimonial. De otra manera los grupos hegemónicos no pueden

35 MARX EN ELSTER, loc cit en nota 9.

36 Marx, El Capital.., vol. I, pág. 55.

37 VICENT, Juan, «Early Social Complexity in Iberia: Some Theoretical Remarks», en Katina T. LiLlos (ed.), The Origins of Complex Societies in Late Prebistoric Iberia, Intemational Monographs in Prehistory, Archaeological Series, 8, Ann Arbor, Michigan, 1995, pp. 177-183.

38 MarX, El Capital..., vol. III, págs. 607-648.

Hi.pania, LVIII/3, núm. 200 (1998) 823-839 
liberarse del carácter contradictorio de su poder y todos sus intentos de consolidarse como señores tributarios estarán condenados al fracaso, como de hecho parecen haber fracasado muchas trayectorias de formación de Estado a lo largo de la Historia, dando lugar a recurrentes «edades oscuras». Se podría decir, por lo tanto, que cada contexto histórico genera un cierto "nivel crítico» de la acumulación originaria, a partir del cuál se hacen viables las oportunidades de los "grandes hombres» patrimoniales de convertirse en señores tributarios imponiendo a los campesinos y a sus propios pares «costes de rebelión» imposibles de asumir y, al mismo tiempo, dejando de depender de la reciprocidad parental para fundamentar sus reclamaciones sobre los medios de producción (incluyendo el trabajo campesino).

La acumulación originaria tributaria plantearía, en tales casos, una nueva situación de «contradicción entre el desarrollo de las fuerzas productivas y las condiciones sociales de la producción', que no es más que un desarrollo de lo que más arriba hemos llamado "contradicción fundamental» del modo de apropiación patrimonial. Esta puede ser, a gtandes rasgos, la «revolución» que echábamos en falta más arriba, descrita en términos suficientemente generales como para incluir los procesos de formación de Estado de la «Revolución Urbanas y los que se dan en contexto agrarios. En cualquier caso, puede ser útil retener y desarrollar el concepto de formación germánica, y tal vez, volver a discutir sobre la "comunidad asiática» para dar cuenta de la evidente diversidad de formas que adopta este «evento central en el desarrollo de la Humanidad» que tan poca atención ha recibido en los debates teóricos.

\section{AGRADECIMIENTOS}

El texto ha mejorado considerablemente gracias a los comentarios críticos de $\mathrm{M}^{2}$ Isabel Martínez Navarrete. Su calidad intelectual, solidaridad y apoyo constante son siempre una premisa insustituible de mi trabajo. Las discusiones con Pedro Díaz del Río a lo largo de la elaboración de su tesis doctoral han contribuído a clarificar mis ideas y me han aportado muchas cosas. Debo agradecerle también su ayuda material en la edición del manuscrito y las notas. Por último, debo reconocer mi deuda intelectual con Antonio Gilman, que va más allá de lo que expresan las referencias explicitas a sus trabajos en el texto. 